\title{
A Distinct Element Method for Analysis of Structures Under Blast Loading
}

\author{
X. B. $\mathrm{Lu}^{1}$ and Y. Y. Guo ${ }^{2}$
}

\begin{abstract}
A three dimensional distinct element method (DEM) for numerical analysis of structural responses such as rolled concrete dam (RCD) under blast loading is presented in this paper. It is shown that the presented DEM is suitable for structural analysis by the comparison of numerical and experimental results of a RCD. The effects of some factors on the numerical results are investigated.
\end{abstract}

Keywords: Blast loading, vibration, DEM.

\section{Introduction}

The RCD is built by rolling concrete layer by layer. Typically, each layer has a thickness of $30 \mathrm{~cm}$. The method based on continuum mechanics can not simulate the main characteristics, while the distinct element method is suitable for the analysis in these conditions. The DEM was first proposed by Cundall (Cundall 1971) and has been used for different applications (Cundall 1988), such as tunnel and mining engineering. The elements are modeled as balls first. Other types of elements ( for example, three-dimensional hexahedron and planar tetrahedron elements.) have been presented to solve different practical problems later (Guo et al. 2000, Dowding et al.1983, Belytschko et al. 1984, Dowding and Gilbert 1988), such as the instability analysis of dams, slopes and tunnels.

In this paper, a modified three dimensional DEM is presented to simulate a structure under blast loading. The elements are contacted with each other in a face-toface way. Each element is taken as a rigid hexahedron. It is shown that DEM is suitable for studying a structure with joints or cracks under blast loading by the comparison of numerical and experimental results.

1 Institute of Mechanics, Chinese Academy of Sciences, 100080, Beijing, China, Email:xblu@imech.ac.cn

2 School of Science, Beihang Univ., 100083, Beijing, China. 


\section{The Face-to-Face Model of Dynamic DEM}

The basic equations. The DEM models a structure as an assemblage of rigid hexahedron connected with each other by springs. Each contact point may bear normal compressive and tangential forces. Each face of one element has four points. The four points are the centers of four small quadrangles with an equal area on each face. The material is considered satisfying the Mohr-Coulumb criterion and cannot bear tensile force. The movement of each element's center satisfies the following equations:

$$
\begin{gathered}
M \ddot{U}_{i}+\xi \dot{U}_{i}+K U_{i}=\sum_{j=1}^{24} F_{i j} \\
F_{i j}=k_{i} \Delta U_{i j} ; \quad K=\sum k_{i}
\end{gathered}
$$

in which $\mathrm{i}$ is the element number. $\mathrm{M}$ is the mass or moment of inertia, $U$ is the displacement or rotate angle surrounding the center, $\mathrm{F}$ is the total force or total moment, $\xi$ is the damping coefficient, $k_{i}$ is the equivalent stiffness and can be determined by the method presented by Goodman and Taylor (1968), $\Delta U_{i j}$ is the difference of displacements between i LUMP and the adjacent LUMP $\mathrm{j}$.

The relation between force and displacement. The forces on each NUMP are connected with the displacements by linear relations as fellows:

$$
\Delta F_{n}=k_{n} \Delta u_{n} ; \Delta F_{s}=k_{s} \Delta u_{s}
$$

in which $\Delta u_{n}, \Delta u_{s}$ are the increments of normal and tangential displacements, respectively, $\Delta F_{n}, \Delta F_{s}$ are the increments of normal and tangential forces, respectively.

If $F_{n}$ equals zero, two contact points are separated. If $F_{s}$ is largger than or equals $C+F_{n} \operatorname{tg} \varphi$, there is a sliding displacement between two contact points $(C, \varphi$ are the cohesion and the angle of internal friction, respectively.).

\section{The Constitutive Relation of the Blast Induced Gas}

A gas sphere with high pressure is assumed to form immediately after blast. The pressure pushes the adjacent elements moving outwards. It is assumed here that there is no thermo-exchange between the gas and the adjacent elements. The state equation is expressed as follows (Rosengren, 1993):

$$
\begin{array}{ll}
P V^{\gamma_{1}}=P_{0} V_{0}^{\gamma_{1}} & P \geq P_{k} \\
P V^{\gamma_{2}}=P_{k} V_{k}^{\gamma_{2}} & P<P_{k}
\end{array}
$$


in which $\gamma_{1}, \gamma_{2}$ are paramaters and equal $\gamma_{1}=3, \gamma_{2}=4 / 3$, respectively, $\mathrm{P}, \mathrm{V}$ are the pressure and volume at any time, $P_{0}, V_{0}$ are the initial pressure and volume, and $P_{k}, V_{k}$ are the critical pressure and volume of the gas sphere, $P_{k}$ is determined by the following equation:

$$
P_{k}=P_{0}\left[\frac{\gamma_{2}-1}{\gamma_{1}-\gamma_{2}}\left(\frac{\left(\gamma_{1}-1\right) Q_{w}}{P_{0} V}-1\right)\right]^{\frac{\gamma_{1}}{\gamma_{1}-1}}
$$

in which $Q_{w}$ is the blast induced heat. $P_{0}$ is determined by the following equation:

$$
P_{0}=\frac{\rho_{w} D^{2}}{2(\gamma+1)}
$$

in which the density $\rho_{w}$ and explosion velocity D of detonator are $1000 \mathrm{~kg} / \mathrm{m}$ and $4000 \mathrm{~m} / \mathrm{s}$, respectively.

\section{The Method for Solving the Momentum Equations}

The dynamic relaxation method is used here to solve the momentum equations (1) and (2). This method is an explicit difference method and each element is assumed to influence the contact elements only. The momentum equations are rewritten as follows in the difference format (Munjiza et al. 1992, Kim et al. 1997, Goodman and Taylor 1968):

$$
\begin{gathered}
m_{i}\left(\dot{u}_{i}^{t}-\dot{u}_{i}^{t-\Delta t}\right)=\Delta t\left\{\left(F_{i}^{e x t}\right)^{t}-\left(F_{i}^{\mathrm{int}}\right)^{t}-\xi_{i} \dot{u}_{i}^{t-\Delta t}\right\} \\
\Delta u_{i}^{t}=\Delta \dot{u}_{i}^{t} \cdot \Delta t
\end{gathered}
$$

in which $\mathrm{i}$ is the element number, $\dot{u}_{i}, u_{i}$ are the velocity and displacement, respectively, $m_{i}$ is the mass of the element, $\Delta t$ is the time increment, $F^{e x t}$ is the external force, $F^{\text {int }}$ is the contact force between two contact elements.

The program in this paper is a modified version of Gao (1999) by adding the ability to simulate blast loading. The development of the blast induced gas sphere and its interaction with the surrounding elements are considered.

The movement of elements near the detonator. The computation includes two stages: In the first stage, the computation is processed under gravity and static loadings; in the second stage, the computation is processed immediately after blast. The blast element (where the detonation is located) keeps integrity in the first stage 
while it is divided into 24 small elements in the second stage and each small element has only one LUMP connected with the adjacent element. The movement of each small element is determined by the following equation:

$$
\left(\ddot{u}_{i}\right)^{\prime}=\frac{1}{m_{i}}\left[\left(F_{i}^{b}\right)^{t}-\left(F_{i}^{i n}\right)^{t}-\xi_{i}\left(\dot{u}_{i}\right)^{t-\Delta t}\right]
$$

in which $\mathrm{i}$ is the small element number, $\ddot{u}_{i}, \dot{u}_{i}, u_{i}$ are the acceleration, velocity and displacement, respectively, $m_{i}$ is the mass, $F_{i}^{b}$ is the blast induced force, $F_{i}^{\text {in }}$ is the force between contact points, $\xi_{i}$ is the damping coefficient.

The comparison of numerical and experimental results. In order to test the reliability of the method and program presented in this paper, the comparison of numerical and experimental results of a RCD is processed. The RCD view is shown in Fig. 1. The dam has a length of $165 \mathrm{~m}$ and a height of $17 \mathrm{~m}$.

The modulus of elasticity and the Poisson's ratio are equal to $5.9 \times 10^{9} \mathrm{~Pa}$ and 0.2 according to the experiments in the literature (Ding et al. 2001, Li et al. 2001). The charge of detonator is $6 \mathrm{~kg}$. The damping coefficient is 0.03 . The dam is divided into an assemblage of rigid hexahedron. The size of each hexahedron is length $\times$ width $\times$ height $=2 \times 2 \times 2 \mathrm{~m}^{3}$. The numerical blast induced gas pressure according to equations (4)-(7) is shown in Fig. 2. It is shown that the pressure increases immediately after blast and then decreases fast, which is similar to the experimental results (Fang 2001). The typical shock lasts for about $50 \mathrm{~ms}$. The other parameters are adopted as follows: $\rho=2400 \mathrm{~kg} / \mathrm{m}^{3}, x_{1}=110 \mathrm{~m}, y_{1}=4 \mathrm{~m}, z_{1}=10 \mathrm{~m}$.

Initial conditions are as follows: $t=0: U=0, V=0, W=0, \sigma_{z}=\rho g z$, $\sigma_{x}=\sigma_{y}=\mu \rho g z /(1-\mu)$, in which $\rho$ is the density of dam, $\left(x_{1}, y_{1}, z_{1}\right)$ is the location of detonator, $(U, V, W)$ and $\left(\sigma_{x}, \sigma_{y}, \sigma_{z}\right)$ are the displacements and the stresses of each element in three directions, respectively.

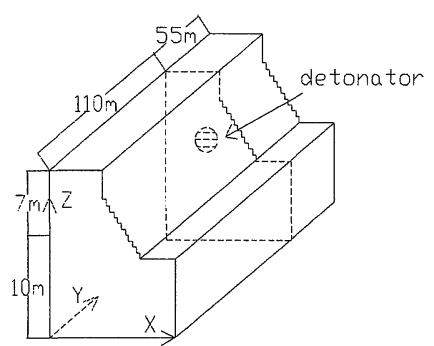

(a) Three dimensional view

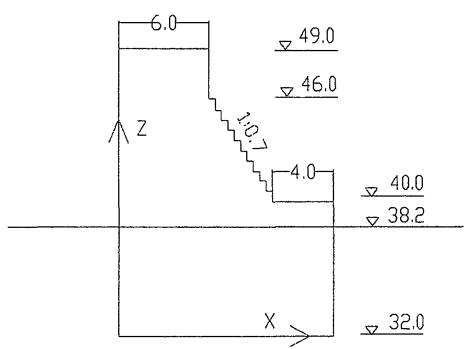

(b) Side view

Fig. 1 The view of the RCD 
Boundary conditions: the bottom is normally fixed. The side surfaces perpendicular to $y$ axis satisfies radiation conditions. The forces on these surfaces are modified by adding a force of $\rho C v_{i}$, in which $\rho, C, v_{i}$ are the density, wave velocity and the velocities in three directions respectively. The surfaces perpendicular to $\mathrm{x}$ axis are normally fixed below the altitude of $38.2 \mathrm{~m}$. The other surfaces are free.

The numerical and experimental results that the vertical velocity degrades with distance are shown in Fig. 3. The data on the center line of the upper surface are chosen here. The degradation of vertical velocity with time is shown in Fig. 4. It is shown that the numerical and experimental results are agreement with each other well.

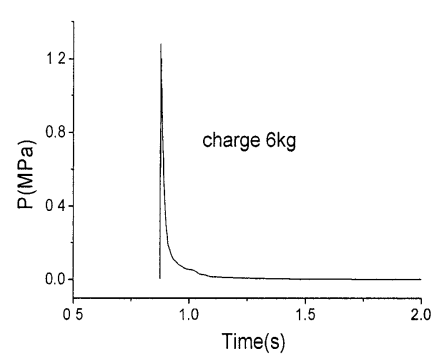

Fig. 2 The numerical blast induced Pressure-time curve

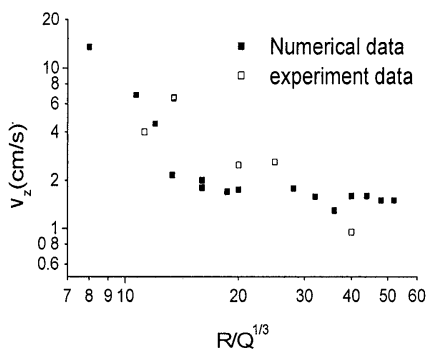

Fig. 3 The comparison of degradation of vertical velocity with distance

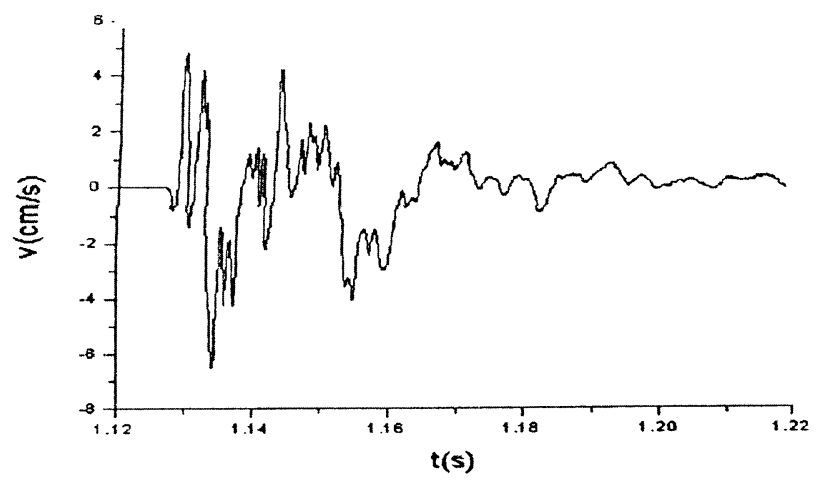

(a) Experiment data on $19 \mathrm{~m}$

Fig. 4 The comparison of degradation of vertical velocity with time (Adopted from Ding et al., 2001; Fang, 2001.) 


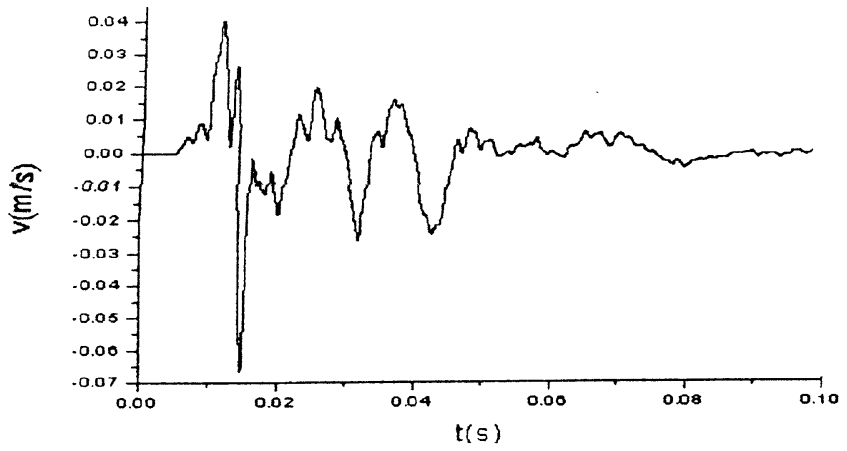

(b) Numerical data on $20 \mathrm{~m}$

Fig. 4. The comparison of degradation of vertical velocity with time (continued)

\section{The Effect of Damping Coefficient and Block Size}

The effects of damping coefficient are shown in Fig. 5. In this figure, the degradation of vertical velocity $V_{z m}$ with $R / Q^{1 / 3}$ (the so called proportional distance in China.) under different damping coefficients is given. The effects of block size are shown in Fig. 6. In this figure, the degradation of vertical velocity with time under different block sizes is given. It is shown that the maximum of velocity decreases with the increase of damping coefficient or with the increase of block size. It may be explained by the fact that the dissipative energy increases with the increase of damping coefficient and block size, which leads to the decrease of vibration and dissipative time.

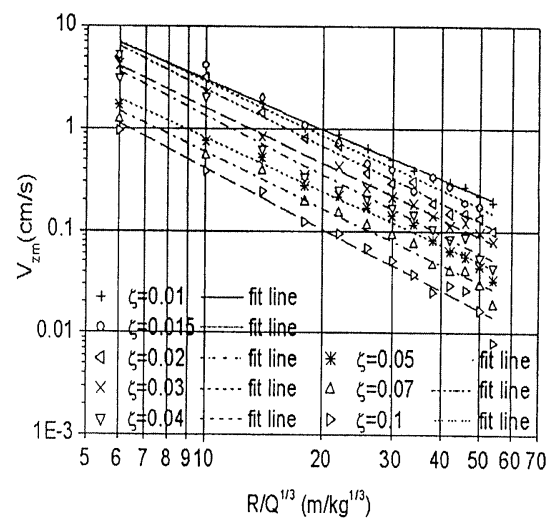

Fig. 5. Effects of damping coefficient 


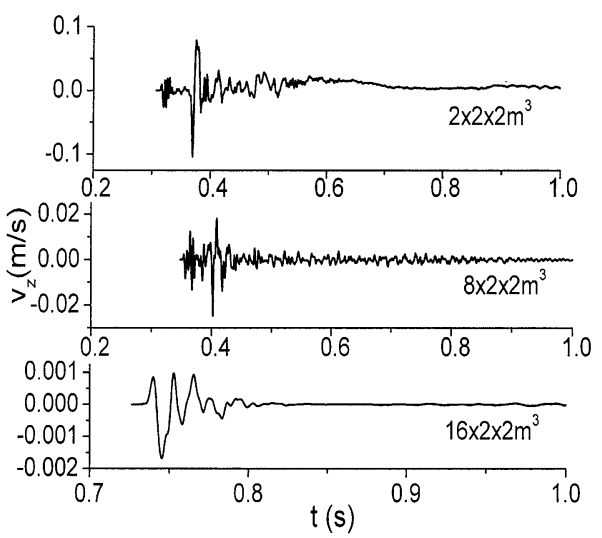

Fig. 6. Effects of block size

\section{Conclusions}

A three dimensional face-to-face DEM is used in the analysis of a RCD under blast loading. The comparison of the responses of a RCD under blast loading is carried out. Some effects of factors are discussed. The main conclusions are as follows: (1) The numerical and experimental results are agreement with each other well, which means, the DEM can simulate the responses of a structure well. (2) The vibration velocity degrades with distance and with time fast. (3) The block size and the damping coefficient influence the responses obviously. The vibration velocity and the dissipative time decrease with the increase of block size and damping coefficient.

\section{References}

Belytschko T., Plesha M., and Dowding C. H. (1984). "A computer method for the stability analysis of caverns in jointed rock." Int. J. Num. Anal. Meth. Geomech, 473492.

Cundall P. A. (1971). "A computer model for simulating progressive large scale movements in elementy systems." Proceedings of the Symposium of the International Society of Rock Mechanics. Nancy, France, 1, Paper No. II-8.

Cundall P. A. (1988). "Formulation of a three-dimensional distinct element modelpart I: a scheme to detect and represent contacts in a system composed of many polyhedral elements." Int. J. Rock Mech. Min. Sci. \& Geomech. Abstr., 25(3), 107116. 
Ding H. K., Fang Z., Li S. H., and Zhang X. H. (2001). "The experiment on the blast probability of RCC." Reseach report of institute of mechanics, Chinese Academy of Sciences.

Dowding C. H., Belytschko T. B.,and Yen H. J. (1983). "Dynamic computational analysis of opening in joint rock." Journal of Geotechnical Engineering, ASCE, 109(12), 1551-156.

Dowding C. H., and Gilbert C. (1988). "Dynamic stability of rock slopes and high frequency traveling waves." Journal of Geotechnical Engineering, ASCE, 114(10), 1069-1088.

Fang Z.(2001). "Experimental and Numerical Simulation of the Demolishing by Blast of a RCC Dam." Thesis for Master Degree, Beijing: Institute of Mechanics, Chinese Academy of Sciences.

Guo Y. Y., Li S. H., and Liu C. T. (2000). "The application of three dimension DEM in the blast of geotechnical engineering." Blast, 17( Appl. ), 19-23.(in Chinese)

Goodman R E, Taylor R L. (1968). "A model for the mechanics of joint rock." Journal of the Soil Mechanics and Foundations Division, ASCE, 94(3), 637-659.

Gao B. (1999). "Modified Three Dimensional Distinct Method and Its Application in the High Slope in The Three Gorge's Strobe Engineering." Thesis for Master Degree, Beijing: Institute of Mechanics, Chinese Academy of Sciences.

Kim M. K., Kim S. E., OH K.H. et al. (1997). "A study on the behavior of rock mass subjected to blast using modified distinct element method." International Journal of Rock Mechanics and Mining Sciences, 34(3/4), 269.

Li S. H, Cheng L., and Fang Z. (2001). "The experiment on the transforming rules of RCC. " Research Report of Institute of Mechanics, Chinese academy of sciences.

Munjiza, A., Owen, D. and Roger J. (1992). "Discrete element models of rock blast." International Journal for Engineering Modelling. 5(3-4), 65-72.

Rosengren, L. (1993). "Preliminary analysis of the dynamic interaction between Norra Länken and a subway tunnel for Stockholm, Sweden". Tunnelling and Underground Space Technology. 8, 429-439. 